\title{
CONTINUITY OF MULTIPLICATION IN OPERATOR ALGEBRAS
}

\section{ALEXANDER BLAIR}

Parts of Banach algebra theory have been generalized recently $[2 ; 1]$ to multiplicatively convex topological algebras. An important class of examples of Banach algebras is the class of subalgebras of the algebra of continuous linear transformations on a Banach space. It is natural to ask whether an algebra of continuous linear transformations on a convex vector space, with the topology of bounded convergence (the analogue of the norm topology in an algebra of transformations on a Banach space), is a multiplicatively convex topological algebra. We show that this is in general false, in fact that if the algebra is large enough multiplication is not even continuous unless the convex vector space is normable.

Theorem. If $X$ is a Hausdorff convex vector space and multiplication is continuous in the topology of bounded convergence in an algebra $A$ of continuous linear transformations on $X$ including the subalgebra of transformations with finite-dimensional range, then $X$ is normable.

Proof. A fundamental system of neighborhoods of the origin in the topology of bounded convergence in $A$ is the family of sets $T(A, V)$ of continuous linear transformations $u$ in $A$ such that $u(A) \subseteq V$, for $A$ a bounded set in $X$ and $V$ a neighborhood of the origin in $X$. Let $V \neq X$ be an equilibrated $(\lambda V \subseteq V$ for $|\lambda|=1)$ neighborhood of the origin in $X$ and let $A \neq 0$ be a bounded set in $X$. Then by the continuity of multiplication in $A$ at the origin there exists a bounded set $A^{\prime}$ in $X$ and a neighborhood $V^{\prime}$ of the origin in $X$ such that $\left[T\left(A^{\prime}, V^{\prime}\right)\right]^{2} \subseteq T(A, V) . V^{\prime}$ may be taken to be equilibrated.

We show there exists a scalar $\lambda \neq 0$ such that $\lambda V^{\prime} \subseteq \bigcup_{u \in T\left(A^{\prime}, V^{\prime}\right)} u(A)$. Let $x_{0} \neq 0$ be an element of $A$. By the Hahn-Banach theorem there exists a continuous linear functional $l_{1}$ on $X$ with $l_{1}\left(x_{0}\right) \neq 0 . l_{1}\left(A^{\prime}\right)$ is bounded. Let $c=\sup _{x \in A^{\prime}}\left|l_{1}(x)\right|$. Let $\lambda=\left(l_{1}\left(x_{0}\right)\right) / c$. Then $\lambda \neq 0$. Given $x$ in $V^{\prime}$ let $\phi_{x}$ be an isomorphism of the scalar field $F$ onto the 1-dimensional subspace $[x]$ generated by $x$. Let $\lambda_{x}=\phi_{x}^{-1}(x)$. Then $\sup _{x \in A^{\prime}}\left|\left(\lambda_{x} l_{1}(x)\right) / c\right|=\left|\lambda_{x}\right|$, so $\left(\lambda_{x} l_{1}\left(A^{\prime}\right)\right) / c \subseteq \phi_{x}^{-1}\left(V^{\prime} \cap[x]\right)$ since $\phi_{x}^{-1}\left(V^{\prime} \cap[x]\right)$ is an equilibrated set in $F$ containing $\phi_{x}^{-1}(x)$. Let $u_{x}$ $=\left(\phi_{x} \lambda_{x} l_{1}\right) / c$. Then $u_{x}$ is in $T\left(A^{\prime}, V^{\prime}\right)$ since $u_{x}\left(A^{\prime}\right)=\left(\phi_{x} \lambda_{x} l_{1}\left(A^{\prime}\right)\right) / c$ $\subseteq V^{\prime} \cap[x] \subseteq V^{\prime}$. Now $\lambda x=u_{x}\left(x_{0}\right)$ for all $x$ in $V^{\prime}$, since $l_{1}\left(x_{0}\right)=\lambda c$, hence $\left(\lambda x l_{1}\left(x_{0}\right)\right) / c=\lambda \lambda_{x}=\lambda \phi_{x}^{-1}(x)$ and hence $u_{x}\left(x_{0}\right)=\left(\phi_{x} \lambda_{x} l_{1}\left(x_{0}\right)\right) / c=\lambda x$.

Received by the editors June 14, 1954.' 
Hence $\lambda V^{\prime} \subseteq \bigcup_{x \in V^{\prime}} u_{x}\left(x_{0}\right) \subseteq U_{u \in T\left(A^{\prime}, V^{\prime}\right)} u(A)$.

We show $V^{\prime}$ is bounded. If $V^{\prime}$ is not bounded then $\lambda V^{\prime}$ is also not bounded, and so there exists a continuous linear functional $l_{2}$ on $X$ not bounded on $\lambda V^{\prime}$. Let $y$ be an element of $X$ not in $V$. Since $V$ is equilibrated $\lambda y \notin V$ if $|\lambda|>1$. Let $\phi$ be an isomorphism of $F$ onto $[y]$. Then $\phi l_{2}$ is a continuous linear transformation of $X$ into $X$, hence $\phi l_{2}\left(A^{\prime}\right)$ is bounded, hence absorbed by $V^{\prime}$, hence there exists $\lambda_{1}>0$ so $\lambda_{1} \phi l_{2} \in T\left(A^{\prime}, V^{\prime}\right)$. Let $u_{2}=\lambda_{1} \phi l_{2} . u_{2} u_{x} \in\left[T\left(A^{\prime}, V^{\prime}\right)\right]^{2} \subseteq T(A, V)$, but $u_{2}\left(\lambda V^{\prime}\right) \subseteq U_{x \in V^{\prime}} u_{2} u_{x}(A)$ and $u_{2}\left(\lambda V^{\prime}\right)$ is an unbounded subset of $[y]$, hence not included in $V$, a contradiction. Hence $V^{\prime}$ is bounded. Since $X$ has a bounded neighborhood $V^{\prime}$ of the origin, $X$ is a normable space, q.e.d.

It is easy to extend the proof to the case where $X$ is not Hausdorff.

\section{BIBLIOGRAPHY}

1. R. F. Arens, A generalization of normed rings, Pacific Journal of Mathematics vol. 2 (1952) pp. 455-471.

2. E. A. Michael, Locally multiplicatively-convex topological algebras, The Memoirs of the American Mathematical Society, no. 11, 1952.

HARVARD UNIVERSITY 